\title{
Motivação para aprender: relação com o desempenho de estudantes ${ }^{1}$
}

\author{
Rita da Penha Campos Zenorini \\ Acácia Aparecida Angeli dos Santos ${ }^{2}$ \\ Rebecca de Magalhães Monteiro \\ Universidade São Francisco, Itatiba-SP, Brasil
}

\begin{abstract}
Resumo: O presente estudo objetivou comparar as orientações de metas de estudantes com alto e baixo desempenho e identificar diferenças relativas às variáveis sexo e tipo de escola. Participaram 110 estudantes dos três anos do ensino médio com idades variando entre 14 e 17 anos provenientes de escola particular e pública do interior de São Paulo. O instrumento utilizado foi a Escala de Avaliação da Motivação para Aprendizagem - EMAPRE. Os resultados apontaram os alunos indicados com alto desempenho como mais orientados à meta aprender e os com baixo desempenho como os mais orientados à meta performance-evitação. Na comparação entre metas e sexo, verificou-se que as meninas apresentaram pontuação média significativamente mais alta na meta aprender. Em relação ao tipo de escola, os alunos da escola pública apresentaram médias significativamente superiores na meta aprender. Esses achados corroboram outros da literatura e evidenciam a necessidade de novas pesquisas.
\end{abstract}

Palavras-chave: motivação, rendimento escolar, avaliação educacional, estudantes de $2^{\circ}$ grau.

\section{Motivation for learning: the relation with students performance}

\begin{abstract}
This study objective was to compare the achievement goals of students with high and low performance and to identify the differences related to gender and type of school. The participants consisted of 110 students from public and private high schools in Sao Paulo with ages ranging from 14 to 17. The instrument used was the Brazilian Scale for Assessment of Motivation for Learning - EMAPRE. The results showed that students with high performance were more oriented to the learning goal and students with low performance were more oriented to the performance avoidance goal. Comparing goals in relation to gender, the findings showed that the girls achieved significantly higher average scores on the learning goal. In relation to the type of school, public school students presented significantly higher scores on the learning goal than private school students. These findings corroborate others in the literature and gives evidence of the need for further research.
\end{abstract}

Keywords: motivation, academic achievement, educational evaluation, high school students.

\section{Motivación para aprender: relación con el desempeño de estudiantes}

Resumen: El objetivo de este trabajo fue comparar orientaciones de metas de estudiantes con desempeño alto y bajo e identificar diferencias relativas a las variables sexo y tipo de escuela. Participaron 110 estudiantes de enseñanza media, 14 a 17 años, de una escuela privada y otra pública del interior de São Paulo. Se utilizó la Escala de Avaliação da Motivação para Aprendizagem - EMAPRE. Los resultados mostraron los alumnos con desempeño alto más orientados a la meta aprender, y los alumnos con desempeño bajo más orientados a la meta performance-evitación. Comparando metas y sexo, se verificó que las niñas presentaron puntuaciones promedio significativamente más altas en la meta aprender. En relación al la escuela, los alumnos de escuela pública presentaron promedios significativamente superiores en la meta aprender. Esos resultados corroboran otros encontrados por la literatura, y muestran la necesidad de nuevas investigaciones.

Palabras clave: motivación, rendimiento escolar, evaluación educacional, estudiantes de $2^{\circ}$ grado.

É inegável que os problemas motivacionais podem interferir na aprendizagem dos estudantes. Muitos estudos têm demonstrado a relação entre o sucesso acadêmico e a motivação (Bzuneck, 2002, 2005). São muitas as variáveis que podem interferir na motivação do estudante, o que a torna um fenômeno bastante complexo. Entre elas, destacam-se o

\footnotetext{
${ }^{1}$ Este texto foi revisado seguindo o Acordo Ortográfico da Língua Portuguesa (1990), em vigor a partir de $1^{\circ}$ de janeiro de 2009 . O artigo apresenta um recorte da pesquisa de campo da tese de doutorado da primeira autora.

${ }^{2}$ Endereço para correspondência:

Acácia Aparecida Angeli Santos. Universidade São Francisco, campus Itatiba. Programa de Pós-graduação Stricto Sensu em Psicologia. R. Alexandre Rodrigues Barbosa, 45. CEP 13.251-900. Itatiba-SP, Brasil. E-mail: acacia.angeli@gmail.com
}

ambiente da sala de aula, as ações do professor, os aspectos emocionais, as questões relacionadas à falta de envolvimento do aluno com situações de aprendizagem, o uso inadequado de estratégias de aprendizagem, entre outras (Guimarães, 2003; Ruiz, 2005, Stefano, 2002; Zenorini \& Santos, 2003, entre outros).

As pesquisas sobre motivação e desempenho demonstram que essa é uma questão que tem despertado a preocupação de educadores e estudiosos. Um dos modelos teóricos adotados por pesquisadores é a Teoria de Metas de Realização, que tem trazido contribuições para o entendimento dos fatores motivacionais que influenciam o comportamento do aluno, pois busca explicar a motivação para aprender, focalizando o aspecto qualitativo do envolvimento desse em seu processo de aprendizagem. Com base na abordagem, os estudiosos entendem 
que as metas ou propósitos que a pessoa deseja atingir estão entre os mais potentes motivadores do comportamento humano (Ames, 1992; Bzuneck, 2002, 2004).

No campo educacional, os estudos com base nessa teoria têm buscado compreender como os estudantes pensam em si próprios, nas suas tarefas e no seu desempenho (Darnon, Harackiewicz, Butera, Mugny, \& Quiamzade, 2007; Gouveia, Diniz, Gouveia, \& Cavalcante, 2008; entre outros). Esses pesquisadores consideram que o tipo de orientação de meta predominante no aluno interfere na maneira como ele trata as tarefas escolares.

Ames (1992) definiu as "metas de realização" como um conjunto de pensamentos, crenças, propósitos e emoções que traduzem as expectativas dos alunos em relação a determinadas tarefas as quais deverão executar, ou seja, as metas são representadas por modos diferentes de enfrentar as tarefas acadêmicas. Os estudos iniciais da teoria apresentam duas metas com características distintas, identificadas em alunos orientados à meta aprender e à meta performance. As terminologias variam de acordo com os pesquisadores: a meta aprender também é denominada de tarefa e domínio; a meta performance-aproximação, de habilidade-aproximação e de ego de automelhora e a meta performance-evitação, de habilidade-evitação e de ego de autoderrota. No presente trabalho, as terminologias utilizadas serão meta aprender e meta performance-aproximação e evitação.

Autores como Middleton e Midgley (1997) observam que a meta aprender tem sido positivamente relacionada ao esforço, à persistência acadêmica, às percepções de eficácia acadêmica, à aprendizagem autorregulada, ao desejo de buscar ajuda para as tarefas de escola e também à motivação intrínseca. Para explicar essas associações positivas, Elliot (1999) sugere que devido ao fato de a meta aprender estar voltada para o desenvolvimento da competência, a luta para se conquistar esses objetivos é fundamentalmente estimulada e baseada em desafios. Assim sendo, presume-se que a sua adoção traz à tona processos comportamentais, cognitivos e afetivos que elevam o número de resultados positivos.

$\mathrm{O}$ aluno orientado à meta performance é caracterizado como aquele que crê que a sua capacidade é evidenciada por "fazer melhor que os outros" e, sendo assim, ele valoriza o reconhecimento público de ser mais capaz do que os demais, proporcionado pela comparação social. O padrão motivacional do aluno orientado à meta performance se caracteriza por contínuas tentativas de demonstrar sua capacidade e competência e também de evitar juízos negativos sobre si mesmo (Ames, 1992; Bzuneck, 2002, 2004; Givvin, 2001; entre outros).

Várias das pesquisas realizadas, principalmente as iniciais, apontam para os efeitos prejudiciais da meta performance (Archer, 1994; Grant \& Dweck, 2003; Midgley, Kaplan, \& Middleton, 2001, entre outros). Contudo, há estudos mais recentes que descobriram efeitos positivos nela, especialmente quando acompanhada da orientação à meta aprender (Bouffard, Boisvert, Vezeau, \& Larouche, 1995;
Harackiewicz, Barron, Pintrich, Elliot, \& Thrash, 2002; entre outros). Há também estudos que apontam esta meta associada tanto a aspectos positivos como negativos, a saber: motivos para alcançar o sucesso como para evitar o fracasso, menores níveis de ansiedade e altos níveis de depressão (Boruchovitch \& Costa, 2004; Dickson \& MacLeod, 2004; Tanaka, Okuno, \& Yamauchi, 2002). Esses estudos apontam dois componentes independentes na meta performance: o de aproximação (buscar parecer inteligente ou de ser o primeiro) e o de evitação (não querer parecer incapaz).

No que se refere ao desempenho escolar, estudos como o de Elliot, McGregor e Gable,(1999) realizados com universitários, encontrou a meta aprender e a meta performance-aproximação correlacionadas positivamente com o desempenho, e a meta performance-evitação, negativamente com o desempenho. Outros estudos que serão apresentados a seguir também evidenciaram tal relação (Harackiewicz, Barron, Tauer, \& Elliot, 2002; Pintrich, 2000).

No estudo longitudinal de Pintrich, realizado com alunos de oitava e nona séries de uma escola secundária, foi investigado como se desenvolviam as tendências motivacionais e as orientações às metas com relação à disciplina de Matemática. Os dados foram coletados em momentos diferentes do ano letivo e, com base neles, os alunos foram classificados em quatro grupos: alunos com escores altos nas metas aprender e performance-aproximação; alunos com escores baixos nas duas metas; alunos com escores altos na meta aprender e baixos na meta performance-aproximação e alunos com escores baixos na meta aprender e altos na meta perfomance-aproximação. Os resultados indicaram efeitos positivos sobre as variáveis crenças, emoções, estratégias e desempenho nos grupos de alunos com altos escores nos dois tipos de metas e dos com altos escores na meta aprender e baixos na performance-aproximação. Esses resultados demonstram o aspecto positivo da meta aprender quando associada à meta performance-aproximação.

Outro estudo longitudinal, realizado por Harackiewicz, Barron, Tauer e cols. (2002), com o objetivo de prever o sucesso no curso superior, utilizou as metas de realização e medidas de habilidade como indicadores de interesse e desempenho de alunos do primeiro ao último ano do curso de graduação. Numa primeira etapa, houve o acompanhamento dos estudantes numa disciplina de psicologia para que se identificassem quais variáveis previam o interesse e o desempenho deles. Os autores identificaram que as metas de realização, além do desempenho no ensino médio, foram preditivos dos resultados iniciais e de longo prazo, sendo que a meta aprender previu o interesse, ao passo que a meta performance-aproximação previu o desempenho. O desempenho no ensino médio previu o desempenho acadêmico, mas não o interesse. As descobertas mais uma vez confirmaram a importância da perspectiva de múltiplas metas.

Entre os estudos brasileiros, identificou-se o de Gouveia e cols. (2008) o qual correlacionou a pontuação obtida no Questionário de Metas de Realização com indicadores de 
desempenho escolar. Os participantes foram 307 estudantes do ensino médio de duas escolas públicas (68\%) e uma particular (32\%) da cidade de João Pessoa-PB, com idade média de 17,6 anos e a maioria do sexo feminino $(61,2 \%)$. Os resultados apontaram a que as metas de aprendizagemaproximação caracterizaram a maioria dos participantes e suas pontuações foram positivamente correlacionadas com o desempenho escolar. Os autores, além de fazerem uma ressalva em relação à consistência interna das dimensões do instrumento, chamaram a atenção para a falta de unanimidade em relação aos resultados que obtiveram, insistindo na importância de mais pesquisas a respeito.

$\mathrm{Na}$ literatura brasileira recuperada, foi identificado um estudo recente, desenvolvido por Martinelli e Genari (2009), o qual investigou especificamente a relação da motivação com o desempenho escolar de 150 alunos do ensino fundamental. Os instrumentos utilizados foram a prova de rendimento escolar da Secretaria de Educação do Estado de São Paulo (SARESP) e uma escala de avaliação da motivação escolar, desenvolvida com base na teoria sociocognitiva. As autoras constataram que tanto na $3^{\text {a }}$, como na $4^{\mathrm{a}}$ série a motivação extrínseca correlacionou-se negativa e significativamente com o desempenho escolar, ou seja, quanto menor o desempenho maior a motivação extrínseca. Os achados do estudo foram congruentes com a literatura na área, embora as autoras afirmem que há pouca evidência empírica a respeito.

Vale ressaltar que, embora o estudo Martinelli e Genari não tenha se baseado na Teoria de Metas de Realização, o estudante motivado à meta aprender assemelha-se ao estudante com motivação intrínseca. No entanto, esses construtos não são idênticos, pois seguem pressupostos diferentes. A motivação intrínseca caracteriza-se pela escolha autodeterminada da ação, sem obrigação externa; o comprometimento com uma atividade é considerado espontâneo e parte do interesse individual do aluno. Na meta aprender, o fator mais importante está no ambiente da sala de aula, e os professores são considerados os principais agentes de seu desenvolvimento, por meio de suas práticas, pela maneira de estruturarem as tarefas, pelo procedimento de avaliação da aprendizagem e pelo feedback, pelo posicionamento diante da classe, entre outros fatores. As características do ambiente, percebidas pelo aluno, exercem forte influência no desenvolvimento de metas. Mesmo assim, as mesmas práticas educativas que promovem a meta aprender podem conduzir simultaneamente, para o desenvolvimento da motivação intrínseca (Bzuneck, 2004).

Assim como o desempenho, a diferença entre os sexos também é uma questão que vem sendo explorada nos estudos que abordam a Teoria de Metas de Realização. Na pesquisa longitudinal de Anderman e Anderman (1999), realizada com estudantes de ensino fundamental em transição para o ensino médio, os autores encontraram os meninos mais orientados à meta performance do que as meninas. $\mathrm{O}$ mesmo foi encontrado nos estudos de Middleton e Midgley (1997), Midgley e cols. (2001) e Zenorini e Santos (2003), os dois primeiros realizados com estudantes do ensino médio e o último, com estudantes universitários.

Os estudos descritos apontam para a existência de relação entre a motivação e o desempenho escolar. Aqueles que adotam a Teoria de Metas de Realização indicam que há diferentes metas associadas a padrões distintos na aprendizagem dos estudantes. Assim, tem sido constatado que os estudantes orientados à meta aprender e à meta performance-aproximação têm demonstrado padrões de aprendizagem mais adaptativos, diferentemente dos orientados à meta performance-evitação.

Considerando o exposto, o presente estudo teve como objetivo comparar as orientações de metas de estudantes indicados como tendo alto e baixo desempenho escolar. Adicionalmente, procurou-se também identificar eventuais diferenças entres as variáveis sexo e tipo de escola.

\section{Método}

\section{Participantes}

Participaram do estudo 110 estudantes, que foram divididos em dois grupos: um de alunos com alto desempenho e outro de alunos com baixo desempenho. Para formação dos grupos, solicitou-se a quatro professores de escolas diferentes, uma particular e três públicas, que indicassem alunos que atendiam aos perfis definidos. Para o primeiro grupo, foi pedido que designassem aqueles alunos que tinham alto rendimento na maioria das disciplinas e que, além disso, mostrassem-se frequentemente interessados nas atividades escolares. Para a formação do segundo grupo, solicitou-se que os professores apontassem os alunos que tinham rendimento escolar baixo na maior parte das disciplinas e que, também, fossem predominantemente desinteressados pelas atividades escolares.

Dessa forma, o grupo de alunos com alto desempenho ficou composto de 58 participantes, sendo 26 meninos e 32 meninas, entre 14 e 17 anos, sendo 14 deles alunos do $1^{\circ}$ ano, 33 do $2^{\circ}$ ano e 11 do $3^{\circ}$ ano. O grupo de alunos com baixo desempenho ficou composto por 52 participantes, 44 deles meninos e 8 meninas, com idade variando entre 15 e 19 anos, sendo 33 alunos do $1^{\circ}$ ano, três do $2^{\circ}$ ano e 16 do $3^{\circ}$ ano.

\section{Instrumento}

O instrumento utilizado no presente trabalho foi a Escala de Avaliação da Motivação para a Aprendizagem (EMAPRE), composta por 50 itens, 20 referentes à meta aprender, 20 à performance-aproximação e 10 à performance-evitação. Os itens referem-se à motivação e às atitudes em relação à aprendizagem estruturados em uma escala Likert e envolvendo três opções de resposta: discordo (1 ponto), não sei (2 pontos) e concordo (3 pontos). A pontuação mínima é de 50 pontos e a máxima de 150 pontos. 
O instrumento apresenta evidência de validade baseada na análise da estrutura interna dos itens por meio de análise fatorial exploratória (Zenorini \& Santos, 2010). Foi identificado, então, a existência de três fatores, correspondentes às três subescalas previstas teoricamente. A consistência interna dos itens das três subescalas foi medida pelo alfa de Cronbach. Para a escala total o $\alpha$ foi de 0,73 , para o Fator 1 (meta aprender), o $\alpha$ foi de 0,80; para o Fator 2 (meta performance-aproximação), de 0,76 e para o Fator 3 (meta performance-evitação) de 0,73.

Para análise da estabilidade temporal da escala, Zenorini e Santos (2010) usaram a técnica do teste-reteste com 77 alunos, realizada após dois meses da primeira aplicação. Os resultados foram analisados pela prova de correlação de Pearson e indicaram que as médias obtidas na segunda aplicação foram muito próximas da primeira. As autoras ressaltam que foi mantida a ordem das médias dos valores para cada meta, sendo a mais elevada a da meta aprender, seguida pela meta performance-aproximação e, por último, a meta performance-evitação.

\section{Procedimento}

Coleta de dados

Nos dias e horários agendados pela direção das escolas, os instrumentos foram distribuídos, tendo sido feita uma coleta de dados coletiva em situação de sala de aula. O tempo de preenchimento foi em torno de 25 minutos, sendo muito boa a adesão dos participantes, visto que houve menos de
$10 \%$ que não responderam ao instrumento, seja pela falta de retorno do Termo de Consentimento Livre e Esclarecido (TCLE) assinado ou pela não disposição do aluno de preencher a escala.

\section{Análise dos dados}

Considerando-se a característica dos dados, optou-se pela prova $U$ de Mann-Whitney da estatística não-paramétrica, a qual não envolve o cálculo de médias dos pontos, mas sim a média dos pontos resultantes do ordenamento dos escores obtidos.

\section{Considerações éticas}

Primeiramente, foi solicitada autorização à direção das escolas para a realização da pesquisa. Após a aprovação do Comitê de Ética em Pesquisa da Universidade São Francisco (Protocolo n ${ }^{\circ} 178 / 05$ ), os participantes foram contatados pelas pesquisadoras e informados sobre os objetivos do estudo e sobre a exigência da assinatura do Termo de Consentimento Livre e Esclarecido pelo responsável, já que os alunos eram menores de idade.

\section{Resultados}

Os resultados obtidos foram organizados seguindo a ordem dos objetivos. Assim, apresenta-se em primeiro lugar a comparação entre os alunos indicados pelos professores como tendo alto desempenho e baixo desempenho. Os resultados são apresentados na Tabela 1 .

Tabela 1

Comparação entre as médias por ranking entre os alunos com alto e baixo desempenho

\begin{tabular}{llcccc}
\hline \multicolumn{1}{c}{ Subescalas } & \multicolumn{1}{c}{ Classificação } & $\boldsymbol{n}$ & Média & $\boldsymbol{z}$ & \multicolumn{1}{c}{$\boldsymbol{p}$} \\
\hline Meta aprender & Alto desempenho & 56 & 63,63 & $-3,608$ & $<0,001$ \\
& Baixo desempenho & 50 & 42,16 & \\
Meta performance-aproximação & Alto desempenho & 58 & 54,00 & $-0,179$ & 0,858 \\
& Baixo desempenho & 50 & 55,08 & \\
Meta performance-evitação & Alto desempenho & 58 & 45,34 & $-3,205$ \\
& Baixo desempenho & 49 & 64,26 & 0,001 \\
\hline
\end{tabular}

Observa-se que, no que se refere à meta aprender, o grupo com alto desempenho obteve uma média por rank significativamente superior. Já com relação à meta performance-evitação, foi o grupo com baixo desempenho que apresentou a média ranqueada significativamente mais alta.
Outro aspecto investigado foi o relativo à diferença entre os sexos, sendo também utilizada a prova $U$ de Mann-Whitney para a comparação proposta. Os resultados podem ser visualizados na Tabela 2. 
Tabela 2

Comparação entre as médias por ranking entre os alunos dos sexos masculino e feminino

\begin{tabular}{|c|c|c|c|c|c|}
\hline Subescala & Sexo & $n$ & Média & $z$ & $p$ \\
\hline \multirow[t]{2}{*}{ Meta aprender } & Masculino & 68 & 48,15 & $-2,411$ & 0,016 \\
\hline & Feminino & 38 & 63,08 & & \\
\hline \multirow[t]{2}{*}{ Meta performance-aproximação } & Masculino & 70 & 56,18 & $-0,759$ & 0,448 \\
\hline & Feminino & 38 & 51,41 & & \\
\hline \multirow[t]{2}{*}{ Meta performance-evitação } & Masculino & 69 & 58,54 & $-2,078$ & 0,038 \\
\hline & Feminino & 38 & 45,76 & & \\
\hline
\end{tabular}

Os resultados indicaram que, quando se comparou a meta aprender, as meninas obtiveram pontuações significativamente superiores. Contudo, na comparação da meta performance-evitação, ocorreu o contrário: os meninos obtiveram pontuações significativamente mais elevadas. No que se refere à meta performance-aproximação, não houve diferenças significativas entre os sexos, tal como na Tabela 3.

Tabela 3

Comparação entre as médias por ranking entre os alunos das escolas pública e particular

\begin{tabular}{lllccc}
\hline \multicolumn{1}{c}{ Subescala } & & Escola & $\boldsymbol{n}$ & Média & $\boldsymbol{z}$ \\
\hline Meta aprender & Pública & 80 & 61,34 & $-4,633$ & $<0,001$ \\
& Particular & 26 & 29,37 & $-0,628$ & 0,530 \\
Meta performance-aproximação & Pública & 82 & 55,56 & 51,15 & \\
& Particular & 26 & 56,64 & $-1,547$ \\
Meta performance-evitação & Pública & 80 & 0,122 \\
& Particular & 27 & 46,17 & \\
\hline
\end{tabular}

Pela análise dos resultados, observou-se que, na comparação da meta aprender, os alunos da escola pública tiveram pontuações significativamente superiores. Contudo, na comparação das metas performance-aproximação e performance-evitação, não houve diferenças significativas entre os dois tipos de escola.

\section{Discussão}

Antes da discussão dos resultados, propriamente dita, vale lembrar que a abordagem teórica adotada neste estudo foi a Teoria de Metas de Realização, que vem contribuindo para a busca do entendimento dos fatores motivacionais que influenciam o comportamento humano, procurando explicar as metas ou os propósitos dos alunos ao se envolverem em uma tarefa de aprendizagem. Nesse sentido, as pesquisas realizadas na área educacional, as quais adotam essa teoria, buscam compreender como os estudantes se veem ante às tarefas escolares e ao seu desempenho.

Os resultados aqui trazidos apontaram os alunos indicados com alto desempenho como mais orientados à meta aprender e os com baixo desempenho como mais orientados à meta performance-evitação. Esses achados vêm confirmar o que tem sido assinalado nos estudos sobre a meta aprender, relacionando-a ao esforço, à persistência, à aprendizagem autorregulada, entre outros aspectos que levam a resultados positivos. O mesmo acontece com os estudos que relacionam a performance-evitação a processos negativos como o processamento superficial da informação, a desorganização, os comportamentos de evitação, a ansiedade, a baixa autoestima e ao baixo desempenho (Elliot, 1999; Middleton \& Midgley, 1997). Nesse sentido, pode-se afirmar que os achados do presente estudo fornecem uma evidência de validade de critério para a EMAPRE, haja vista o fato de que a média dos escores obtidos pelos alunos com bom e mau rendimento, conforme a categorização realizada pelo professor, é congruente com os aspectos teóricos que fundamentaram a construção da escala.

A comparação entre meninos e meninas, aqui realizada, indicou que as meninas obtiveram pontuações médias superiores na meta aprender, enquanto os meninos pontuaram significativamente mais na meta performance-evitação. Esses resultados vão ao encontro dos achados nos estudos de Middleton e Midgley (1997) que constataram que as meninas afro-americanas apresentam maior tendência à meta aprender, atitudes mais positivas em sala de aula e maior autoeficácia e os meninos, às metas performance-aproximação e evitação. Na mesma direção dos achados do presente 
estudo, foi o resultado de Zenorini e Santos (2003) com uma amostra de universitários. As autoras também identificaram as participantes do sexo feminino como mais orientadas à meta aprender.

No presente estudo, não houve diferenças entre os sexos em relação à meta performance-aproximação. Contrariamente, alguns estudos como os Anderman e Anderman (1999) e Midgley e cols. (2001), além do de Middleton e Midgley (1997), já mencionado, encontraram os meninos mais orientados à meta performance-aproximação do que as meninas. De modo geral, as pesquisas descrevem os alunos orientados a esse tipo de meta como aqueles preocupados com a comparação social, com a demonstração de habilidades superiores e com o interesse pela a aprovação de outros. Dentre os aspectos apontados como negativos, destaca-se que, muitas vezes, o aluno pode limitar-se à recompensa $\mathrm{e}$ à satisfação, e a ausência dessas oportunidades pode levar à frustração, ao desanimo e à falta de persistência.

No entanto, há autores que destacam o papel positivo da meta performance, principalmente em seu componente aproximação. Entre eles, estão Bouffard e cols. (1995), Harackiewicz, Barron, Pintrich e cols. (2002), Harackiewicz, Barron, Tauer e cols. (2002). Há também aqueles que apontam aspectos positivos e negativos, tais como Dickson e MacLeod (2004) e Tanaka e cols. (2002).

O componente evitação da meta performance se apresenta constantemente relacionado a aspectos negativos como a baixa autodeterminação, o baixo desempenho, a ansiedade ante as avaliações, a pouca ou nenhuma persistência. Esses alunos têm medo de correr riscos, isolam-se e não buscam ajuda (Elliot \& cols., 1999; Middleton \& Midgley, 1997).

O resultado da comparação das metas dos alunos das escolas pública e particular foi curioso e incita à realização de mais pesquisas para explorar o seu significado. Na comparação das metas performance-aproximação e performanceevitação, não houve diferenças significativas entre os dois tipos de escola. Contudo, no que se refere à meta aprender, houve diferença favorável e estatisticamente significativa aos alunos da escola pública.

Importante destacar que, nas pesquisas recuperadas para a realização deste estudo, verificou-se que os resultados relativos à meta aprender são os que apresentam maior consistência nos resultados. O aluno orientado a este tipo de meta é destacado como aquele que demonstra maior envolvimento, interesse e prazer no processo de aprendizagem, enfrentando tarefas desafiadoras e não desanimando diante do fracasso. Assim, mostra-se mais persistente e se envolve mais ativamente nas atividades escolares.

\section{Considerações finais}

Ao realizar a pesquisa, com estudantes do ensino médio, buscou-se fornecer indicadores para melhor compreender a motivação para a aprendizagem de alunos que se encontram em uma fase bastante peculiar. É sabido que essa fase é comumente marcada por frustrações, temores, dúvidas, expectativas, construção de valores, em que se acentua a busca da formação da identidade, a qual vai depender das circunstâncias sociais, culturais e históricas, que poderão facilitar ou dificultar esse processo.

Outra consideração interessante diz respeito ao fato de que predominantemente as pesquisas focalizam um nível específico de ensino. Ressalta-se a importância de estudos longitudinais, para verificar como se dá a motivação no decorrer da vida acadêmica. Esses aspectos sugerem a realização de novas pesquisas, que permitam uma melhor avaliação dos efeitos das orientações de metas nos alunos; para tanto poderiam também ser avaliadas a relação das metas com outras variáveis como estratégias de aprendizagem, senso de participação escolar, crenças motivacionais, autoeficácia, persistência, esforço, ansiedade, desempenho acadêmico, entre outras.

Cabe aqui destacar as implicações educacionais dessas avaliações, na medida em que a Teoria de Metas de Realização enfatiza fatores como o ambiente da sala de aula e o professor, o qual, por meio da sua prática, pode ser o agente propulsor que irá auxiliar o desenvolvimento das metas. Para isso, faz-se necessário implementar práticas educativas que busquem uma relação dialógica, em que a discussão e o debate sejam valorizados, assim como, a importância do envolvimento, da participação e do esforço de cada aluno.

É importante considerar que são inúmeros os problemas enfrentados no sistema educacional brasileiro, principalmente na rede pública, como a falta de professores com formação adequada, a infraestrutura precária, as metodologias utilizadas, o sistema avaliativo e, no caso específico do ensino médio, o dilema que gira em torno de preparar o aluno com o enfoque para o trabalho ou para o ensino superior. Também não se pode deixar de mencionar a deficiência decorrente do ensino fundamental, a qual gera uma grande heterogeneidade com relação ao nível dos alunos, pois, assim como há aqueles que chegam ao ensino médio com uma boa base, há aqueles que chegam minimamente alfabetizados.

Diante desse quadro, faz-se necessário que tanto o professor como aluno se envolvam com o processo de ensino e aprendizagem e que se valorize o processo e não o produto da aprendizagem. É certo que o aluno deseja tirar boas notas, e isso é importante, pois a sociedade e o mercado de trabalho assim o exigem. No entanto, não se pode reduzir o processo de ensino e aprendizagem apenas à "cultura das boas notas", já que nem sempre a boa nota revela a verdadeira aprendizagem.

Assim, mais uma vez, evidencia-se a necessidade de investimentos em pesquisas, discussões e estudos que proponham alternativas para auxiliar os educadores a pensar em mecanismos para uma aprendizagem mais efetiva dos seus alunos, ou seja, uma aprendizagem que envolva a dedicação, o esforço, a persistência, o desafio, visando à autonomia do sujeito. Pode-se dizer que os resultados encontrados neste estudo foram, em grande parte, coerentes com os relatados na produção recuperada sobre o tema. 
A par das limitações relativas ao tamanho da amostra e à desigualdade das subamostras (sexo e tipo de escola), entende-se que o estudo acrescentou algumas informações ao pouco que se sabe sobre os alunos do ensino médio e como se caracterizam em relação ao perfil motivacional. Outras indagações permanecem, especialmente às relativas ao fato de haver mais meninos no grupo considerado pelos professores como de baixo desempenho. Embora não tenha sido esse o foco do estudo aqui apresentado, ele pode suscitar inquietação suficiente para que pesquisadores se debrucem sobre a questão investigando o quanto há aí de mito ou realidade.

\section{Referências}

Ames, C. (1992). Classrooms: Goals, structures, and student motivation. Journal of Education Psychology, 84(3), 261-271.

Anderman, H. L., \& Anderman, E. M. (1999). Social predictors of changes in students' achievement goal orientations. Contemporary Educational Psychology, 24(1), 21-37.

Archer, J. (1994). Achievement goals as a measure of motivation in university students. Contemporary Educational Psychology, 19(4), 430-446.

Bouffard, T., Boisvert, J., Vezeau, C., \& Larouche, C. (1995). The impact of goal orientation on self-regulation and performance among college students. British Journal of Educational Psychology, 65(3), 317-329.

Boruchovitch, E., \& Costa, E. R. (2004). O impacto da ansiedade no rendimento escolar e na motivação de alunos. In E. Boruchovitch \& J. A. Bzuneck (Orgs.), A motivação do aluno: Contribuições da psicologia contemporânea (pp. 134-147). Petrópolis, RJ: Vozes.

Bzuneck, J. A. (2004). A motivação do aluno: Orientado a metas de realização. In E. Boruchovitch \& J. A. Bzuneck (Orgs.), A motivação do aluno: Contribuições da psicologia contemporânea (pp. 58-77). Petrópolis, RJ: Vozes.

Bzuneck, J. A. (2005). A motivação dos alunos em cursos superiores. In M. C. R. A. Joly, A. A. A. Santos, \& F. F. Sisto (Orgs.), Questões do cotidiano universitário (pp. 217-237). São Paulo: Casa do Psicólogo.

Darnon, C., Harackiewicz, J. M., Butera, F., Mugny, G., \& Quiamzade, A. (2007). Performance-approach and performance-avoidance goals: When uncertainty makes a difference. Personality and Social Psychology Bulletin, 33(6), 813-827.

Dickson, J. M., \& MacLeod, A. K. (2004). Anxiety, depression and approach and avoidance goals. Cognition and Emotion, 18(3), 423-430.

Elliot, A. J. (1999). Approach and avoidance motivation and achievement goals. Educational Psychologist, 34(3), 169-189.
Elliot, J. A., McGregor, H. A., \& Gable, S. (1999). Achievement goals, study strategies, and exam performance: A mediational analysis. Journal of Educational Psychology, 91(3), 549-563.

Givvin, K. B. (2001). Goal orientations of adolescents, coaches, and parents: Is there a convergence of beliefs? Journal of Early Adolescence, 21(2), 227-247.

Gouveia, V. V., Diniz, P. K. C., Santos, W. S., Gouveia, R. S. V., \& Cavalcanti, J. P. N. (2008). Metas de realização entre estudantes do ensino médio: Evidências de validade fatorial e consistência interna de uma medida. Psicologia: Teoria e Pesquisa, 24(4), 535-544.

Grant, H., \& Dweck, C. S. (2003). Clarifying achievement goals and their impact. Journal of Personality and Social Psychology, 85(3), 541-554.

Guimarães, S. E. R. (2003). Avaliação do estilo motivacional do professor: Adaptação e validação de um instrumento. Tese de doutorado não-publicada, Universidade Estadual de Campinas, Campinas, SP.

Harackiewicz, J. M., Barron, K. E., Pintrich, P. R., Elliot, A. J., \& Thrash, T. M. (2002). Revision of achievement goal theory: Necessary and illuminating. Journal of Educational Psychology, 94(3), 638-645.

Harackiewicz, J. M., Barron, K. E., Tauer, J. M., \& Elliot, A. J. (2002). Predicting success in college: A longitudinal study of achievement goals and ability measures as predictors of interest and performance from freshman year through graduation. Journal of Educational Psychology, 94(3), 562-575.

Martinelli, S. C., \& Genari, C. H. M. (2009). Relações entre desempenho escolar e orientações motivacionais. Estudos de Psicologia (Natal), 14(1), 13-21.

Middleton, M. J., \& Midgley, C. (1997). Avoiding the demonstration of lack of ability: An underexplored aspect of goal theory. Journal of Education Psychology, 89(4), 710-718.

Midgley, C., Kaplan, A., \& Middleton, M. (2001). Performance-approach goals: Good for what, for whom, under what circumstances, and at what cost? Journal of Educational Psychology, 93(1), 77-86.

Pintrich, P. R. (2000). Multiple goals, multiple pathways: The role of goal orientation in learning and achievement. Journal of Educational Psychology, 92(3), 544-555.

Ruiz, V. M. (2005). Aprendizagem em universitários: Variáveis motivacionais. Tese de doutorado nãopublicada, Pontifícia Universidade Católica, Campinas, SP.

Stefano, S. R. (2002). As orientações motivacionais em cursos de Administração: Um estudo comparativo entre alunos de instituição pública e de instituição privada. Dissertação de mestrado não-publicada, Universidade Estadual de Londrina, Londrina, PR. 
Tanaka, A., Okuno, T., \& Yamauchi, H. (2002). Achievement motives, cognitive and social competence, and achievement goals in the classroom. Perceptual and Motor Skills, 95(2), 445-458.

Zenorini, R. P. C., \& Santos, A. A. A. (2003). A motivação e a utilização de estratégias de aprendizagem em universitários. In E. Mercuri \& S. A. J. Polydoro (Orgs.), Estudante universitário: Característica e experiências de formação (pp. 67-86). Taubaté, SP: Cabral Editora e Livraria Universitária.

Zenorini, R. P. C., \& Santos, A. A. A. (2010). Escala de metas de realização como medida da motivação para a aprendizagem. Interamerican Journal of Psychology, 44(2), 291-298.

Rita da Penha Campos Zenorini é Professora Doutora da Anhanguera Educacional.

Acácia Aparecida Angeli dos Santos é Professora Doutora da Universidade São Francisco, campus Itatiba.

Rebecca de Magalhães Monteiro é doutoranda do Programa de Pós-graduação Stricto Sensu da Universidade São Francisco, campus Itatiba.

Recebido: 23/11/2009

$1^{a}$ revisão: $13 / 09 / 2010$

Aceite final: 17/12/2010 